\title{
Effects of different training modalities on phosphate homeostasis and local vitamin D metabolism in rat bone
}

\author{
Joost Buskermolen $^{1}$, Karen van der Meijden $^{2}$, Regula Furrer ${ }^{3}$ ， Dirk-Jan Mons ${ }^{1}$, Huib van Essen ${ }^{1}$, \\ Annemieke Heijboer ${ }^{1}$, Paul Lips ${ }^{2}$, Richard Jaspers ${ }^{3}$, Nathalie Bravenboer ${ }^{\text {Corresp. }}{ }^{1}$ \\ 1 Department of Clinical Chemistry, VU University Medical Center, Amsterdam, The Netherlands \\ 2 Department of Internal Medicine/Endocrinology, VU University Medical Center, Amsterdam, The Netherlands \\ 3 Laboratory for Myology, VU University Amsterdam, Amsterdam, The Netherlands \\ Corresponding Author: Nathalie Bravenboer \\ Email address: n.bravenboer@vumc.nl
}

Objectives: Mechanical loading may be an important factor in the regulation of bone derived hormones involved in phosphate homeostasis. This study investigated the effects of peak power and endurance training on expression levels of fibroblast growth factor 23 (FGF23) and 1 $\alpha$-hydroxylase (CYP27b1) in bone.

Methods: Thirty-eight rats were assigned to six weeks of training in four groups: peak power (PT), endurance (ET), PT followed by ET (PET) or no training (control). In cortical bone, FGF23 was quantified using immunohistochemistry. mRNA expression levels of proteins involved in phosphate and vitamin $D$ homeostasis were quantified in cortical bone and kidney. C-terminal FGF23, 25-hydroxyvitamin D3, parathyroid hormone (PTH), calcium and phosphate concentrations were measured in plasma or serum.

Results: Neither FGF23 mRNA and protein expression levels in cortical bone nor FGF23 plasma concentrations differed between the groups. In cortical bone, mRNA expression levels of sclerostin (SOST), dental matrix protein 1 (DMP1), phosphate-regulating gene with homologies to endopeptidases on the $X$ chromosome (PHEX) and matrix extracellular phosphoglycoprotein (MEPE) were lower after PT compared to ET and PET. Expression levels of CYP27b1 and vitamin D receptor (VDR) in tibial bone were decreased after PT compared to ET. In kidney, no differences between groups were observed for mRNA expression levels of CYP27b1, 24-hydroxylase (CYP24), VDR, NaPi-lla cotransporter (NPT2a) and NaPi-llc cotransporter (NPT2C). Serum PTH concentrations were higher after PT compared to controls.

Conclusion: After six weeks, none of the training modalities induced changes in FGF23 expression levels. However, PT might have caused changes in local phosphate regulation within bone compared to ET and PET. CYP27b1 and VDR expression in bone was reduced after PT compared to ET, suggesting high intensity peak power training in this rat model is associated with decreased vitamin D signalling in bone. 
1 Full Title: Effects of different training modalities on phosphate homeostasis and local

2 vitamin $\mathbf{D}$ metabolism in rat bone.

3

4 Short Title: Effects of training on phosphate homeostasis and vitamin $D$ in bone.

5

6 J Buskermolen ${ }^{1}, \mathrm{~K}$ van der Meijden ${ }^{2}$, R Furrer ${ }^{3}$, D Mons ${ }^{1}$, HW van Essen ${ }^{1}$, AC Heijboer ${ }^{1}$, P

$7 \quad$ Lips $^{2}$, RT Jaspers 3 , N Bravenboer ${ }^{1 *}$

8

$9{ }^{1}$ Department of Clinical Chemistry, VU University Medical Center, Amsterdam Movement

10 Sciences, The Netherlands

11 2Department of Internal Medicine/Endocrinology, VU University Medical Center, Amsterdam

12 Movement Sciences, The Netherlands

$13{ }^{3}$ Laboratory for Myology, Amsterdam Movement Sciences, Faculty of Behavioural and

14 Movement Sciences, Vrije Universiteit Amsterdam, The Netherlands

15

$16 *$ Corresponding author

17 E-mail: n.bravenboer@vumc.nl (NB) 
19

20

21

22

23

24

25

26

27

28

29

30

\section{Abstract}

Objectives: Mechanical loading may be an important factor in the regulation of bone derived hormones involved in phosphate homeostasis. This study investigated the effects of peak power and endurance training on expression levels of fibroblast growth factor 23 (FGF23) and 1 $\alpha$-hydroxylase (CYP27b1) in bone.

Methods: Thirty-eight rats were assigned to six weeks of training in four groups: peak power (PT), endurance (ET), PT followed by ET (PET) or no training (control). In cortical bone, FGF23 was quantified using immunohistochemistry. mRNA expression levels of proteins involved in phosphate and vitamin D homeostasis were quantified in cortical bone and kidney. C-terminal FGF23, 25-hydroxyvitamin D3, parathyroid hormone (PTH), calcium and phosphate concentrations were measured in plasma or serum.

Results: Neither FGF23 mRNA and protein expression levels in cortical bone nor FGF23 plasma concentrations differed between the groups. In cortical bone, mRNA expression levels of sclerostin (SOST), dental matrix protein 1 (DMP1), phosphate-regulating gene with homologies to endopeptidases on the X chromosome (PHEX) and matrix extracellular phosphoglycoprotein (MEPE) were lower after PT compared to ET and PET. Expression levels of CYP27b1 and vitamin D receptor (VDR) in tibial bone were decreased after PT compared to ET. In kidney, no differences between groups were observed for mRNA expression levels of CYP27b1, 24hydroxylase (CYP24), VDR, NaPi-IIa cotransporter (NPT2a) and NaPi-IIc cotransporter (NPT2c). Serum PTH concentrations were higher after PT compared to controls.

Conclusion: After six weeks, none of the training modalities induced changes in FGF23 expression levels. However, PT might have caused changes in local phosphate regulation within bone compared to ET and PET. CYP27b1 and VDR expression in bone was reduced after PT 
43 compared to ET, suggesting high intensity peak power training in this rat model is associated 44 with decreased vitamin D signalling in bone.

45 
46

47

48

49

50

51

52

53

54

55

56

57

58

59

60

61

62

63

64 65

66

67

68

69

\section{Introduction}

Mechanical forces associated with physical exercise cause deformation of bone tissue.

These deformations activate an acute response in osteocytes, in particular production of cytokines and signalling molecules such as nitric oxide (NO), bone morphogenetic proteins (BMPs), Wnts, and prostaglandin E2 (PGE2) (1). These factors affect osteoblast and osteoclast recruitment, differentiation and activation locally at the sites that are subjected to high strains. Since whole bone response to mechanical loading depends on the strain rate and magnitude $(2,3)$, peak power training with high peak loads with a high frequency is generally considered to result in a higher bone response than endurance training. Moreover, it was reported that a combination of training types even better maintained bone mineral density in postmenopausal women (4). Recently, it was proposed that mechanical loading affects gene expression and local protein activity of fibroblast growth factor 23 (FGF23) (5). FGF23 mRNA expression in mouse bone has been shown to increase after 6 days of endurance training (6). Moreover, it was suggested FGF23 might be a 'molecular mediator of the whole-body effects of exercise originating from bone' (7). Indeed, serum FGF23 increased after endurance exercise in rat and human $(8,9)$, indicating a potential systemic effect of local mechano-response in bone; FGF23 is produced exclusively in bone but acts on the kidneys, where it decreases the reabsorption and increases excretion of phosphate and also suppress $1 \alpha$-hydroxylase (CYP27b1), reducing its ability to activate vitamin D and subsequently impairing calcium absorption (10). To our knowledge, the direct effect of different training types on FGF23 expression within bone has not been reported yet.

A known stimulator of FGF23 production is systemic 1,25-dihydroxyvitamin D3 $(1,25(\mathrm{OH}) 2 \mathrm{D} 3)(11)$ or in vitro locally produced 1,25(OH)2D3 (12). Inhibitors of FGF23 
70 production are mineralization-regulating proteins such as phosphate-regulating gene with

71 homologies to endopeptidases on the X chromosome (PHEX) and dental matrix protein 1

72 (DMP1) (13), possibly through alterations in bone mineralization. Mechanical loading could

73 either affect FGF23 gene expression directly, or indirectly by alterations in the expression of

74 FGF23-regulating proteins known to be stimulated by mechanical loading, such as DMP1

$75(14,15)$ and matrix extracellular phosphoglycoprotein (MEPE) (16) as well as presumably locally

76 converted $1,25(\mathrm{OH}) 2 \mathrm{D} 3$.

77

The active metabolite 1,25(OH)2D3 is hydroxylated from 25-hydroxyvitamin D3

78 (25(OH)D3) by $1 \alpha$-hydroxylase (17), which is, among other tissues, expressed by human bone

79 cells $(18,19)$. The regulation of activity of $1 \alpha$-hydroxylase within bone is yet to be determined.

80 Van Driel et al. demonstrated that in cultured bone cells 25(OH)D3, PTH and calcium did not

81 affect CYP27b1 mRNA expression and activity (18). In contrast, treatment of primary human

82 osteoblasts with high doses of calcium did increase CYP27b1 mRNA expression (20). Pulsatile

83 fluid flow, an in vitro model for mechanical loading, increased CYP27b1 expression in primary

84 human osteoblasts (21), suggesting that $1 \alpha$-hydroxylase within bone is at least partly regulated

85 by mechanical loading. Among healthy, young women, serum 25(OH)D3 did not differ between

86 different training groups (22). However, among rats an increase in serum 1,25(OH)2D3 after

87 endurance training compared to controls was observed (23). Whether vitamin D signalling within

88 bone is affected by mechanical loading in vivo remains to be elucidated.

89 Osteocytes may respond to mechanical loading by altering the expression of FGF23 and

90 CYP27b1. As the response of osteocytes to mechanical loading is determined by the type of

91 loading, FGF23 production and $1 \alpha$-hydroxylation may be differentially changed according to the

92 type of loading. We hypothesize that mechanical loading induced by physical exercise influences 
93 FGF23 production and $1 \alpha$-hydroxylation in bone, with the greatest response after a combination

94 of both peak power training and endurance training. Therefore, the aim of this study is to

95 investigate how different kinds of training modalities affect the production of phosphate

96 regulating proteins and $1 \alpha$-hydroxylation by rat bone tissue in vivo. 
98

99

100

101

102

103

104

105

106

107

108

109

110

111

112

113

114

116

117

118

119

120

121

122

123

\section{Materials and Methods}

\section{Experimental design and training protocol}

The animal experiment was approved by the Animal Experiment Committee of the VU University Amsterdam with permit number FBW 10-03, and described previously (24). Briefly, 38 female Wistar rats at the age of 13 weeks were assigned to four groups: control $(n=8)$, peak power training $(\mathrm{PT}, \mathrm{n}=10)$, endurance training $(\mathrm{ET}, \mathrm{n}=10)$ and a combined training $(\mathrm{PET}, \mathrm{n}=10)$. The rats were trained on a treadmill for 6 weeks, 5 days a week for 1 session a day (ET and PT group). For the peak power training, rats performed 10 sprints of $15 \mathrm{sec}$ in gallop at a maximal attainable velocity on a progressively increasing slope starting at $10 \%$ reaching up to $40 \%$ by the end. Endurance training consisted of treadmill running for $10 \mathrm{~min}$ at a speed of $16 \mathrm{~m} / \mathrm{min}$ without a slope which was gradually increased up to $45 \mathrm{~min}$ of length with a speed of $26 \mathrm{~m} / \mathrm{min}$ (trotting) on a $10 \%$ slope. The rats following a combination of training types (PET) conducted 2 training sessions a day: peak power training in the morning and endurance training $8 \mathrm{~h}$ later. Previously, an $8 \mathrm{~h}$ rest period was shown sufficient to restore full mechanosensitivity (25). All rats were sacrificed $22 \mathrm{~h}$ after the last training by cardiac injection with Euthasol 20\% (AST Farma B.V., Oude water, The Netherlands). The animals were group housed, rodent diet (Teklad Global 16\% Protein Rodent Diet, Madison, WI, USA) containing 1\% calcium, 0.7\% phosphorus and $1.5 \mathrm{IU} / \mathrm{g}$ vitamin D3 was provided ad libitum and the rats were kept on a reversed $12 \mathrm{~h}$ light/dark cycle to provide the training during their active period of the day.

\section{Immunohistochemistry}

Immunohistochemistry was performed on the right tibial shaft to analyse protein expression of FGF23. Non-decalcified tibiae were fixed in $4 \%$ phosphate buffered 
124 paraformaldehyde and after dehydration in increasing alcohol series, embedded in $80 \%$

125 methylmethacrylate (MMA) (BDH Chemicals, Poole, England) with 20\% dibuthylphtalate

126 (Merck, Darmstadt, Germany). Longitudinal five-micrometer-thick tissue sections were cut using

127 a Polycut 2500 S microtome (Reichert-Jung, Nussloch, Germany). To remove MMA, tissue

128 sections were incubated in 50\% xylene and 50\% chloroform. Subsequently, sections were

129 rehydrated in a series of decreasing alcohol concentrations. Decalcification was done with $1 \%$

130 acidic acid, followed by quenching of endogenous peroxidases with $3 \% \mathrm{H} 2 \mathrm{O} 2$ in $40 \%$

131 methanol/PBS. Tissue sections were incubated with $10 \%$ goat serum to prevent unspecific

132 binding of the secondary antibody. Subsequently, sections were incubated overnight at $4^{\circ} \mathrm{C}$ with

133 polyclonal 1:200 rabbit anti-FGF23 (AB_2104625, Santa Cruz Biotechnology Sc-50291, CA,

134 USA). The next day, tissue sections were incubated with polyclonal 1:100 biotinylated goat-anti-

135 rabbit (AB_2313609, Dako E0432, Heverlee, Belgium) for $1 \mathrm{~h}$ and with 1:200 horseradish

136 peroxidase labelled streptavidin (Invitrogen, Life Technologies, Bleiswijk, The Netherlands) for

$1371 \mathrm{~h}$. Signal enhancement was established by treatment with tyramide (Invitrogen, Life

138 Technologies, Bleiswijk, The Netherlands) for $10 \mathrm{~min}$ followed by a second $1 \mathrm{~h}$ incubation with

139 1:200 horseradish peroxidase labelled streptavidin. Chromogenesis was performed by treatment

140 of the sections with AEC reagent (Invitrogen, Life Technologies, Bleiswijk, The Netherlands)

141 for 6 min and by counterstaining with haematoxylin. Finally, the sections were mounted with

142 ClearMount Mounting Solution (Invitrogen, Life Technologies, Bleiswijk, The Netherlands) and

143 covered with a coverslip.

144 For each rat, two or three longitudinal tissue sections (depending on the quality of the tissue

145 section), separated by $150 \mu \mathrm{m}$, were analysed using an Olympus BX51 Microscope at 200x

146 magnification. The entire length of the tibial shaft was divided in alternating longitudinal regions 
147 of interest of $50 \mu \mathrm{m}$ long, in which all osteocytes across the entire thickness of the cortex were

148 counted manually. Per tissue section, up to 400 osteocytes were counted. The amount of

149 positively stained osteocytes was compared to the total number of osteocytes and expressed as

150 fraction positively stained osteocytes compared to the total number of osteocytes.

151

\section{Quantitative polymerase chain reaction (qPCR)}

153

154 Tissue preparation and RNA-extraction

155

156

Left tibiae and kidneys were snap frozen and stored at $-80^{\circ} \mathrm{C}$ until further analysis. One

157 week before tibial RNA-isolation and $40 \mathrm{~h}$ prior to kidney RNA-isolation, tibiae and kidneys

158 were stored in RNAlater ${ }^{\circledR}$ - ICE (Ambion, Life Technologies, Bleiswijk, The Netherlands). The

159 kidneys were homogenised using a Dounce Homogeniser (Sigma-Aldrich, Zwijndrecht, The

160 Netherlands). Subsequent processing occurred according to protocol from the column-based

161 'FavorPrep Tissue Total RNA Purification Maxi Kit' (Favorgen Biotech corp., Huissen, The

162 Netherlands). For RNA isolation of the tibiae, proximal and distal ends of the tibiae were cut off.

163 Bone marrow was removed by flushing the diaphysis with ice cold RNAse free water.

164 Diaphyseal cortical bone was pulverised with a freezer-mill (SPEX 6750, Glen Creston,

165 Stanmore, England) and incubated in Trizol (Invitrogen, Life Technologies, Bleiswijk, The

166 Netherlands) for $1 \mathrm{~h}$ at $37^{\circ} \mathrm{C}$. After the first Trizol-extraction, a chloroform-isomyl alcohol

167 extraction was performed, followed by a second Trizol-extraction. Both kidney and tibial RNA

168 were treated with DNAse (Promega, Leiden, The Netherlands) to remove DNA-contamination. 
170

171

172

173

174

175

176

177

178

179

180

181

182

183

184

185

186

187

188

189

190

191

192

193

194

\section{Reversed Transcription}

After RNA-isolation, $100 \mathrm{ng}$ of total RNA was reverse-transcribed using $10 \mathrm{ng} / \mu \mathrm{l}$ random primers (Roche, Almere, The Netherlands) and $5 \mathrm{U} / \mu 1 \mathrm{M}-\mathrm{MLV}$ Reverse Transcriptase in a mixture with $5 \mathrm{mM} \mathrm{MgCl}$, 1 x RT-buffer, $1 \mathrm{mM}$ dNTPs each, $1 \mathrm{M}$ betaine and $0.40 \mathrm{U} / \mu \mathrm{l} \mathrm{RNAsin}$ (Promega, Leiden, The Netherlands). A total volume of $20 \mu \mathrm{l}$ was incubated for $10 \mathrm{~min}$ at $25^{\circ} \mathrm{C}$, $1 \mathrm{~h}$ at $37^{\circ} \mathrm{C}$ and 5 min at $95^{\circ} \mathrm{C}$.

\section{Quantitative PCR}

A total volume of $25 \mu \mathrm{l}$ containing $3 \mu \mathrm{l}$ of cDNA, $1000 \mathrm{nM}$ primers and 12,5 $\mu \mathrm{l} \mathrm{SYBR}$ Green Supermix (Bio-Rad, Veenendaal, The Netherlands) was amplified in the iCycler system (Bio-Rad, Veenendaal, The Netherlands) using the primers as described in Table 1. PCR consisted of an initial denaturation step for $3 \mathrm{~min}$ at $95^{\circ} \mathrm{C}$, followed by 40 amplification cycles $\left(15 \mathrm{sec}\right.$ at $95^{\circ} \mathrm{C}, 1 \mathrm{~min}$ at $\left.60^{\circ} \mathrm{C}\right)$. Subsequently, a melting curve was run from $50^{\circ} \mathrm{C}$ to $95^{\circ} \mathrm{C}$ to check the specificity of the reactions.

The following mRNA levels were assessed in the tibia: 1 $\alpha$-hydroxylase (CYP27b1), 24hydroxylase (CYP24), vitamin D receptor (VDR), fibroblast growth factor 23 (FGF23,) dental matrix protein 1 (DMP1), phosphate-regulating gene with homologies to endopeptidases on the X chromosome (PHEX), matrix extracellular phosphoglycoprotein (MEPE) and sclerostin $(\mathrm{SOST})$.

The following mRNA levels were assessed in the kidney: CYP24, CYP27b1, VDR, NaPi-IIa cotransporter (NPT2a or SLC34a1) and NaPi-IIc cotransporter (NPT2c or SLC34a3). Levels of tibial and kidney mRNA were expressed relative to the average of reference genes hypoxanthine phosphoribosyltransferase (HPRT) and porphobilinogendeaminase (PBGD) 
195 using the $2^{-\Delta \mathrm{CT}}$ method. All samples were assessed in duplicate or triplicate on a single 96 well196 plate per gene.

197

198

199

200

\section{Serum Biochemical Analysis}

201

Blood was obtained by puncture of the vena cava during general anaesthesia prior to sacrifice. Rat C-terminal FGF23 Elisa Kit (Immutopics, San Clemente, CA, USA) was used to 202 measure FGF23 in EDTA plasma. Serum 25(OH)D3 was detected using liquid 203 chromatography - tandem mass spectrometry (LC-MS/MS) according to standardised methods 204 (26). Serum PTH was measured using the Rat Intact PTH Elisa Kit (Immutopics, San Clemente, CA, USA). Serum calcium and phosphate concentrations were measured using an Elecsys platform (Roche Diagnostics, Mannheim, Germany).

\section{Statistical analysis}

GraphPad Prism 6.0 (GraphPad Software Inc., La Jolla, CA, USA) was used for data

211 analysis. Differences between the four groups were tested with a Kruskal-Wallis Test, followed 212 by a Dunn's post-hoc test. A value of $\mathrm{p}<0.05$ was considered to be significant. Results are 213 reported as means per group. Within figures, each dot represents the mean value of a single rat, 214 whereas the bars represent the means per group. 
216 Results

217

218

219

220

\section{Animal experiment}

Of the thirty rats that performed training, three rats (two of the PT group and one of the

221 ET group) were excluded for not fulfilling the required training protocol. This resulted in a total

222 sample size for controls $n=8$, ET $n=9, P T n=8$ and PET $n=10$. The final body weight of the

223 included rats $(\mathrm{n}=35)$ did not differ among the groups. Tibial RNA-isolation for one control rat

224 failed. We could not collect plasma samples for one control, three ET-rats, two PT-rats and one 225 PET-rat.

226

227

228

229

\section{FGF23 protein expression in tibiae}

As shown in figure 1, immunohistochemical analysis of FGF23 protein expression by

230 cortical osteocytes in the tibiae did not reveal any differences in the number of stained cells between the four groups. FGF23 staining in trabecular bone could unfortunately not be quantified, due to low contrast between positive osteocytes and aspecific staining of the marrow.

\section{mRNA expression in tibiae}

Figure 2A shows that FGF23 mRNA expression levels did not differ amongst the groups. mRNA levels of osteocyte maturation markers SOST, DMP1, PHEX and MEPE were significantly lower after PT compared to those after ET and PET, as illustrated by figures 2B-E, respectively. Furthermore, figures $2 \mathrm{~F}$ and $2 \mathrm{G}$ show that CYP27b1 and VDR mRNA expression

240 levels were lower after PT compared to those after ET. Expression of CYP24 mRNA was below 241 detection limit. 


\section{3 mRNA expression in kidneys} mRNA expression levels of FGF23 responsive genes in the kidney to assess whether this would

247 have systemic influences. Figure 3 reveals that there were no significant differences in

249 tissue physical exercise did not induce any changes in mRNA expression levels of FGF23-

250 responsive genes and VDR.

\section{Serum biochemical analysis}

Figure 4 shows there were no significant differences in plasma c-term FGF23

257 levels were not significantly different between groups.

258

259

260

261

262

263

264

265

266

267 
269

270

271

272 phosphate homeostasis and $1 \alpha$-hydroxylation in rat bone in vivo.

273

274

275

276

277

278

279

280

281

282

283

284

285

286

287

288

289

290

291

292

\section{Discussion} measurement.

This study aimed to investigate whether different kinds of training modalities affect

In contrast to our hypothesis, we observed no differences between groups in FGF23 expression in both bone and serum. In line with our results, FGF23 serum levels were not affected by either submaximal exercise or high intensity exercise with a bicycle ergometer among young men (27). Yet, Lombardi et al. described increased FGF23 serum concentrations among participants of the multiple-stage bicycle race Giro d'Italia (9). However, confounding factors in that study could be the high dietary intake as well as weight loss, suggesting the observed changes might be an effect of altered metabolic state rather than mechanical loading of bone cells. Moreover, these cyclists showed signs of induced bone resorption (28), possibly as a consequence of the heavy metabolic stress in the absence of mechanical loading, whereas we assume a situation of bone formation after mechanical loading in our model. Our findings also stand in contrast with those of Li et al., who reported increased serum FGF23 after acute exercise, exhaustive exercise, and chronic exercise among mice (8). However, these mice trained for only one week, whereas the rats in our model performed exercise for up to 6 weeks. As Li et al. also described that FGF23 promotes exercise endurance, it is possible the rats in our study at first responded to training with FGF23-upregulation to adapt to the exercise, but that after 6 weeks the rats were adapted and thus no longer showed increased FGF23 at the time of

Quantification of FGF23 substrates, such as genes involved in vitamin D metabolism and sodium-phosphate co-transporters in kidney tissue, ruled out that post-translational modification differed amongst groups. As FGF23 is considered the major phosphate regulating hormone (10), 
293 it is not surprising that the lack of change in FGF23 or FGF23 substrates was paralleled with

294 unchanged phosphate concentrations in serum after 6 weeks of training. Therefore, these results

295 indicate that FGF23 production in bone and systemic phosphate homeostasis were not affected

296 after 6 weeks of treadmill running.

297 Surprisingly, PT caused a decrease in both CYP27b1 and VDR mRNA levels in tibial

298 bone compared to mRNA levels after ET, suggesting decreased vitamin D signalling in bone in

299 the PT group. We had expected to observe an increase in local vitamin D signalling after PT

300 compared to ET. Possibly, PT might have been too strenuous, causing fatigue damage of the

301 bone leading to apoptosis of osteocytes(29). This process could also have accounted for the

302 observed decreased vitamin D signalling after PT. In line with other studies $(9,22)$, no

303 differences between groups were observed for serum $25(\mathrm{OH}) \mathrm{D}$ concentrations, suggesting the

304 observed decreased vitamin D signalling is rather a local process within bone than a systemic

305 effect of exercise.

306 We hypothesized that alterations in FGF23 expression and $1 \alpha$-hydroxylation in bone

307 tissue in response to mechanical loading are related; both FGF23 expression and $1 \alpha-$

308 hydroxylation are likely to be altered by mechanical loading and both processes may be involved

309 in regulation of each other. As we did not observe differences in FGF23 production but did

310 observe decreased vitamin D signalling in bone tissue, we cannot confirm this hypothesis.

311 Previous studies show contradicting results regarding this issue. In cultured rat osteoblasts

312 FGF23 mRNA expression levels appeared to be enhanced by "autocrine/paracrine action of

313 osteoblast-derived $1 \alpha, 25(\mathrm{OH}) 2 \mathrm{D}$ ” (12). In contrast, incubating cultured primary human

314 osteoblasts with FGF23 did not cause changes in mRNA expression of CYP27b1 and VDR (20).

315 Moreover, in human bone samples an association between FGF23 and CYP27b1, CYP24 or 
316 VDR mRNA expression could not be shown (30). Possibly, in our rat model an acute change in

317 FGF23 expression and 1 $\alpha$-hydroxylation in bone tissue may have occurred, but a new bone

318 balance between the two was established at the time of investigation.

319 Furthermore, PTH levels were higher after PT compared to controls 22 hours after the

320 last training. It is likely that the observed PTH concentrations after PT reflect an increased basal

321 level of PTH in this group after six weeks of training. In general, a transient increase of PTH is

322 observed during and directly after exercise, depending on the type of exercise (31), and most

323 studies report normalization of PTH serum concentrations shortly after exercise (31-33), but

324 some studies observed a prolonged increase in PTH 24 hours after a single exercise (34-36).

325 Low basal PTH concentrations are associated with higher physical fitness (34).

326 Another interesting observation is that PT compared to ET and PET showed lower

327 expression of osteocyte maturation markers SOST, DMP1, PHEX and MEPE. Therefore, it

328 seems that PT caused a suppression of mature osteocyte function, which was reversed by

329 additional endurance training. In general, DMP1 and MEPE are known to increase after

330 mechanical loading (14-16), whereas SOST is downregulated by mechanical loading $(37,38)$.

331 DMP1, MEPE and PHEX are not only markers of osteocyte maturation, but are functionally

332 involved in phosphate regulation and bone mineralization (39). DMP1 and PHEX are known to

333 be involved in inhibition of FGF23 production (13). Therefore, it was expected that reduction of

334 these factors would lead to an increase in FGF23 production in the PT group in our rat model.

335 However, after 6 weeks of peak training FGF23 production and function in systemic phosphate

336 homeostasis appeared unchanged, which does not exclude that local changes in phosphate

337 regulation in bone tissue may have occurred after PT. Moreover, it has been suggested that

338 DMP1 and MEPE cause local changes in stiffness and mineralization of canaliculi and lacunae, 
339 thereby altering the osteocytic response to mechanical loading (40). Possibly a new balance has

340 been established between DMP1, MEPE and FGF23 after 6 weeks of peak training, in which

341 FGF23 carries out its systemic functions whereas DMP1 and MEPE have a local function in

342 lacunar remodelling.

343 Local mechano-response in bone has the potential to lead to systemic effects, because

344 bone is an endocrine organ (41), and changes in bone resorption and formation can lead to

345 alterations in systemic calcium and phosphate. However, in comparison to the many local

346 mechano-responsive changes in tibial bone in this rat model, the only observed systemic effect

347 was an alteration of PTH concentrations after PT. PTH concentrations respond to many other

348 stimuli than just mechanical loading, such as changes in the adrenergic system, $\mathrm{pH}$ and or lactic

349 acid (42). As neither systemic FGF23, 25(OH)D3, calcium and phosphate, nor any of the genes

350 measured in kidney tissue differed between groups, it seems that the effects of mechanical

351 loading in this model did not lead to major systemic differences between groups.

352

Remarkably, we did not observe any statistically significant effect of PET compared to

353 controls or ET. Therefore, it seems that the observed changes in gene and protein expression

354 after PT have been reversed by additional ET. These results should be interpreted with caution;

355 the rats performing PET had more training than the other groups. By performing both training

356 programs, they underwent a higher amount of strain, which might be a confounding factor.

357 In this study organs were harvested 22 hours after the last training session in order to

358 study contractile muscle force characteristics in situ (24). Therefore, acute effects of mechanical

359 loading on mRNA concentrations might have already disappeared at the time of investigation.

360 The observed differences after PT might indicate a possible new steady state. 
362 bone. Trabecular bone is generally considered to be metabolic active and possible effects of

363 exercise on FGF23 protein expression within bone marrow could unfortunately not be quantified. 


\section{Conclusion}

368

369

In conclusion, six weeks of physical exercise did not cause significant differences in

370 phosphate homeostasis on a systemic level. However, mRNA expression levels of genes

371 involved in local phosphate regulation and vitamin D signalling within bone tissue differed

372 among groups, suggesting a local mechano-response rather than a systemic response in this rat 373 model. 


\section{Acknowledgements}

376

377 We would like to thank Ina Kamphuis and Mirjam Bethlehem for their assistance in cutting the

378 tissue sections. Also, we are thankful to the technicians of the Endocrine Laboratory of the VU

379 University Medical Center for the biochemical analyses.

380 
382

383

384

385

386

387

388

389

390

391

392

393

394

395

396

397

398

399

400

401

402

403

404

405

406

407

408

409

410

411

412

413

414

415

416

417

418

419

420

421

422

\section{Reference list}

1. Klein-Nulend J, Bacabac RG, Bakker AD. Mechanical loading and how it affects bone cells: The role of the osteocyte cytoskeleton in maintaining our skeleton. Eur Cells Mater. 2012;24:278-91.

2. Rubin CT, Lanyon LE. Regulation of bone mass by mechanical strain magnitude. Calcif Tissue Int. 1985;37(4):411-7.

3. Turner CH, Owan I, Takano Y. Mechanotransduction in bone: role of strain rate. Am J Physiol. 1995 Sep;269(3 Pt 1):E438-42.

4. Martyn-St James M, Carroll S. A meta-analysis of impact exercise on postmenopausal bone loss: the case for mixed loading exercise programmes. Br J Sports Med. 2009;43:898-908.

5. Sapir-Koren R, Livshits G. Osteocyte control of bone remodeling: is sclerostin a key molecular coordinator of the balanced bone resorption-formation cycles? Osteoporos Int. 2014;25(12):2685-2700.

6. Gardinier JD, Al-Omaishi S, Morris MD, Kohn DH. PTH Signaling Mediates Perlacunar Remodeling During Exercise. Matrix Biol. 2016;(52-54):162-75.

7. Qi Z, Liu W, $\mathrm{Lu}$ J. The mechanisms underlying the beneficial effects of exercise on bone remodeling: Roles of bone-derived cytokines and microRNAs. Prog Biophys Mol Biol. Elsevier Ltd; 2016;122(2):131-9.

8. Li DJ, Fu H, Zhao T, Ni M, Shen FM. Exercise-stimulated FGF23 promotes exercise performance via controlling the excess reactive oxygen species production and enhancing mitochondrial function in skeletal muscle. Metabolism. Elsevier Inc.; 2016;65(5):747-56.

9. Lombardi G, Corsetti R, Lanteri P, Grasso D, Vianello E, Marazzi M, et al. Reciprocal regulation of calcium-/phosphate-regulating hormones in cyclists during the Giro d ' Italia 3-week stage race. Scand J Med Sci Sports. 2014;24:779-87.

10. Bonewald LF, Wacker MJ. FGF23 Production by Osteocytes. Pediatr Nephrol. 2013;28(4):563-8.

11. Saji F, Shigematsu T, Sakaguchi T, Ohya M, Orita H, Maeda Y, et al. Fibroblast growth factor 23 production in bone is directly regulated by $1\{$ alpha\},25-dihydroxyvitamin $\mathrm{D}$, but not PTH. Am J Physiol Renal Physiol. 2010 Nov;299:F1212-7.

12. Tang WJ, Wang LF, Xu XY, Zhou Y, Jin WF, Wang HF, et al. Autocrine/paracrine action of vitamin D on FGF23 expression in cultured rat osteoblasts. Calcif Tissue Int. 2010;86(5):404-10.

13. Martin A, Liu S, David V, Li H, Karydis A, Feng JQ, et al. Bone proteins PHEX and DMP1 regulate fibroblastic growth factor Fgf23 expression in osteocytes through a common pathway involving FGF receptor (FGFR) signaling. FASEB J. 2011;25(8):255162.

14. Gluhak-Heinrich J, Ye L, Bonewald LF, Feng JQ, MacDougall M, Harris SE, et al. Mechanical loading stimulates dentin matrix protein 1 (DMP1) expression in osteocytes in vivo. J bone Miner Res. 2003 May;18(5):807-17. 
423 15. Yang W, Lu Y, Kalajzic I, Guo D, Harris MA, Gluhak-Heinrich J, et al. Dentin matrix

424

425

426

427

428

429

430

431

432

433

434

435

436

437

438

439

440

441

442

443

444

445

446

447

448

449

450

451

452

453

454

455

456

457

458

459

460

461

462

463 protein 1 gene cis-regulation: use in osteocytes to characterize local responses to mechanical loading in vitro and in vivo. J Biol Chem. 2005 May 27;280(21):20680-90.

16. Reijnders CMA, Van Essen HW, Van Rens BTTM, Van Beek JHGM, Ylstra B, Blankenstein MA, et al. Increased expression of matrix extracellular phosphoglycoprotein (MEPE) in cortical bone of the rat tibia after mechanical loading: Identification by oligonucleotide microarray. PLoS One. 2013;8(11):1-12.

17. Lips P. Vitamin D physiology. Prog Biophys Mol Biol. 2006 Sep;92:4-8.

18. van Driel M, Koedam M, Buurman CJ, Hewison M, Chiba H, Uitterlinden AG, et al. Evidence for auto/paracrine actions of vitamin D in bone: 1alpha-hydroxylase expression and activity in human bone cells. FASEB J. 2006 Nov;20:2417-9.

19. van der Meijden K, Lips P, van Driel M, Heijboer AC, Schulten EAJM, den Heijer M, et al. Primary Human Osteoblasts in Response to 25-Hydroxyvitamin D3, 1,25Dihydroxyvitamin D3 and 24R,25-Dihydroxyvitamin D3. PLoS One. 2014;9(10):1-11.

20. van der Meijden K, Essen HW, Bloemers FW, Schulten EAJM, Lips P, Bravenboer N. Regulation of CYP27B1 mRNA Expression in Primary Human Osteoblasts. Calcif Tissue Int. Springer US; 2016;99(2):164-73.

21. van der Meijden K, Bakker AD, van Essen HW, Heijboer AC, Schulten EAJM, Lips P, et al. Mechanical loading and the synthesis of $1,25(\mathrm{OH}) 2 \mathrm{D}$ in primary human osteoblasts. J Steroid Biochem Mol Biol. Elsevier Ltd; 2016;156:32-9.

22. Lester ME, Urso ML, Evans RK, Pierce JR, Spiering BA, Maresh CM, et al. Influence of exercise mode and osteogenic index on bone biomarker responses during short-term physical training. Bone. 2009;45:768-76.

23. Iwamoto J, Shimamura C, Takeda T, Abe H, Ichimura S, Sato Y, et al. Effects of treadmill exercise on bone mass, bone metabolism, and calciotropic hormones in young growing rats. J Bone Miner Metab. 2004;22:26-31.

24. Furrer R, Jaspers RT, Baggerman HL, Bravenboer N, Lips P, de Haan A. Attenuated increase in maximal force of rat medial gastrocnemius muscle after concurrent peak power and endurance training. Biomed Res Int. 2013 Jan;2013:935671.

25. Robling AG, Burr DB, Turner $\mathrm{CH}$. Recovery periods restore mechanosensitivity to dynamically loaded bone. J Exp Biol. 2001;204:3389-99.

26. van der Meijden K, Bravenboer N, Dirks NF, Heijboer AC, den Heijer M, de Wit GMJ, et al. Effects of 1,25(OH)2D3and 25(OH)D3on C2C12 Myoblast Proliferation, Differentiation, and Myotube Hypertrophy. J Cell Physiol. 2016;231(11):2517-28.

27. Emrich IE, Baier M, Zawada AM, Meyer T, Fliser D, Scharhag J, et al. Plasma FGF23 does not rise during physical exercise as a physiological model of sympathetic activation. Clin Res Cardiol. 2018;

28. Lombardi G, Lanteri P, Graziani R, Colombini A, Banfi G, Corsetti R. Bone and Energy Metabolism Parameters in Professional Cyclists during the Giro d'Italia 3-weeks Stage Race. PLoS One. 2012;7(7):e42077.

29. Jilka RL, Noble B, Weinstein RS. Osteocyte apoptosis. Bone. 2013;54(2):264-71. 
464 30. Ormsby RT, Findlay DM, Kogawa M, Anderson PH, Morris HA, Atkins GJ. Analysis of

465

466

467

468

469

470

471

472

473

474

475

476

477

478

479

480

481

482

483

484

485

486

487

488

489

490

491

492

493

494

495

496

497

498

499

500

501

502 vitamin D metabolism gene expression in human bone: Evidence for autocrine control of bone remodelling. J Steroid Biochem Mol Biol. Elsevier Ltd; 2013;144:110-3.

31. Maimoun L, Sultan C. Effect of Physical Activity on Calcium Homeostasis and Calciotropic Hormones : A Review. Calcif Tissue Int. 2009;85:277-86.

32. Scott JPR, Sale C, Greeves JP, Casey A, Dutton J, Fraser WD. The role of exercise intensity in the bone metabolic response to an acute bout of weight-bearing exercise. J Appl Physiol. 2011;110:423-32.

33. Gardinier JD, Mohamed F, Kohn DH. PTH Signaling During Exercise Contributes to Bone Adaptation. J Bone Miner Metab. 2015;30(6):1053-63.

34. Brahm H, Piehl-Aulin K, Ljunghall S. Bone Metabolism During Exercise and Recovery: The Influence of Plasma Volume and Physical Fitness. Calcif Tissue Int. 1997;61:192-8.

35. Bouassida A, Zellag D, Zaouali Ajina M, Gharbi N, Duclos M, Richalet JP, et al. Parathyroid hormone concentrations during and after two periods of high intensity exercise with and without an intervening recovery period. Eur J Apply Physiol. 2003;88:339-44.

36. Thorsen K, Kristoffersson A, Hultdin J, Lorentzon R. Effects of Moderate Endurance Exercise on Calcium, Parathyroid Hormone, and Markers of Bone Metabolism in Young Women. Calcif Tissue Int. 1997;60:16-20.

37. Robling AG, Niziolek PJ, Baldridge LA, Condon KW, Allen MR, Alam I, et al. Mechanical stimulation of bone in vivo reduces osteocyte expression of Sost/sclerostin. J Biol Chem. 2008 Feb 29;283(9):5866-75.

38. Gardinier JD, Al-omaishi S, Morris MD, Kohn DH. PTH signaling mediates perilacunar remodeling during exercise. Matrix Biol. International Society of Matrix Biology; 2016;52-54:162-75.

39. Rowe PSN. Regulation of Bone-Renal Mineral and Energy Metabolism: The PHEX, FGF23, DMP1, MEPE ASARM Pathway. Crit Rev Eukaryot Gene Expres. 2012;22(1):61-86.

40. Harris SE, Gluhak-Heinrich J, Harris MA, Yang W, Bonewald LF, Riha D, et al. DMP1 and MEPE expression are elevated in osteocytes after mechanical loading in vivo: Theoretical role in controlling mineral quality in the perilacunar matrix. J Musculoskelet Neuronal Interact. 2007;7(4):313-5.

41. Han Y, You X, Xing W, Zhang Z, Zou W. Paracrine and endocrine actions of bone - The functions of secretory proteins from osteoblasts, osteocytes, and osteoclasts. Bone Res. Springer US; 2018;6(16):1-11.

42. Bouassida A, Latiri I, Bouassida S, Zalleg D, Zaouali M, Feki Y, et al. Parathyroid hormone and physical exercise: a brief review. J Sports Sci Med. 2006;5:367-74. 
504 Supporting information captions

505

506 Table 1. Details of primers used for quantitative PCR analysis. CYP24: 24-hydroxylase;

507 CYP27b1: 1 $\alpha$-hydroxylase; DMP1: dental matrix protein 1; FGF23: fibroblast growth factor 23;

508 HPRT: hypoxanthine phosphoribosyltransferase; MEPE: matrix extracellular

509 phosphoglycoprotein; NPT2a: NaPi-IIa cotransporter; NPT2c: NaPi-IIc cotransporter; PBGD:

510 porphobilinogendeaminase; PHEX: phosphate-regulating gene with homologies to

511 endopeptidases on the X chromosome; SOST: sclerostin; VDR: vitamin D receptor.

512

513 Figure 1. Effects of different training modalities (ET: endurance training; PT: peak training;

514 PET: combined peak- and endurance training) on the expression of FGF23 in rat tibiae as

515 analysed by immunohistochemistry. (A) Fraction positively stained cortical osteocytes compared

516 to total amount of osteocytes. (B) Representative image of FGF23 positively stained cortical

517 osteocytes after PET. Arrows show positively stained osteocytes, whereas arrowheads point out

518 FGF23 negative osteocytes. Bar represents $25 \mu \mathrm{m}$.

519

520 Figure 2. Effects of different training modalities (ET: endurance training; PT: peak training;

521 PET: combined peak- and endurance training) on mRNA expression in rat tibiae as analysed by

522 qPCR. Gene expression levels of (A) FGF23, (B) SOST, (C) DMP1, (D) PHEX, (E) MEPE, (F)

523 CYP27b1 and (G) VDR were measured. Results were normalized for reference genes HPRT and

524 PBGD. Significant differences are indicated as $* p<0.05$ and $* * p<0.01$.

525

526 Figure 3. Effects of different training modalities (ET: endurance training; PT: peak training;

527 PET: combined peak- and endurance training) on mRNA expression in rat kidneys as analysed 
528 by qPCR. Gene expression levels of (A) CYP27b1, (B) CYP24, (C) VDR, (D) NPT2a and (E)

529 NPT2c were measured. Results were normalized for reference genes HPRT and PBGD.

530 Significant difference is indicated as * $p<0.05$.

531

532 Figure 4. Effects of different training modalities on serum concentrations of factors involved in

533 phosphate homeostasis. Serum concentrations of (A) c-terminal FGF23, (B) 25(OH)D3, (C)

534 parathyroid hormone (PTH), (D) calcium and (E) phosphate were assessed. Significant

535 difference is indicated as ${ }^{*} p<0.05$.

536

537 


\section{Figure 1 (on next page)}

Effects of different training modalities on the expression of FGF23 in rat tibiae as analysed by immunohistochemistry.

(A) Fraction positively stained cortical osteocytes compared to total amount of osteocytes. (B) Representative image of FGF23 positively stained cortical osteocytes after PET. Arrows show positively stained osteocytes, whereas arrowheads point out FGF23 negative osteocytes. Bar represents $25 \mu \mathrm{m}$.

(ET: endurance training; PT: peak training; PET: combined peak- and endurance training) 


\section{Figure 2 (on next page)}

Effects of different training modalities on mRNA expression in rat tibiae as analysed by qPCR.

Gene expression levels of (A) FGF23, (B) SOST, (C) DMP1, (D) PHEX, (E) MEPE, (F) CYP27b1 and (G) VDR were measured. Results were normalized for reference genes HPRT and PBGD. Significant differences are indicated as $* p<0.05$ and ${ }^{* *} p<0.01$.

(ET: endurance training; PT: peak training; PET: combined peak- and endurance training) 
PeerJ
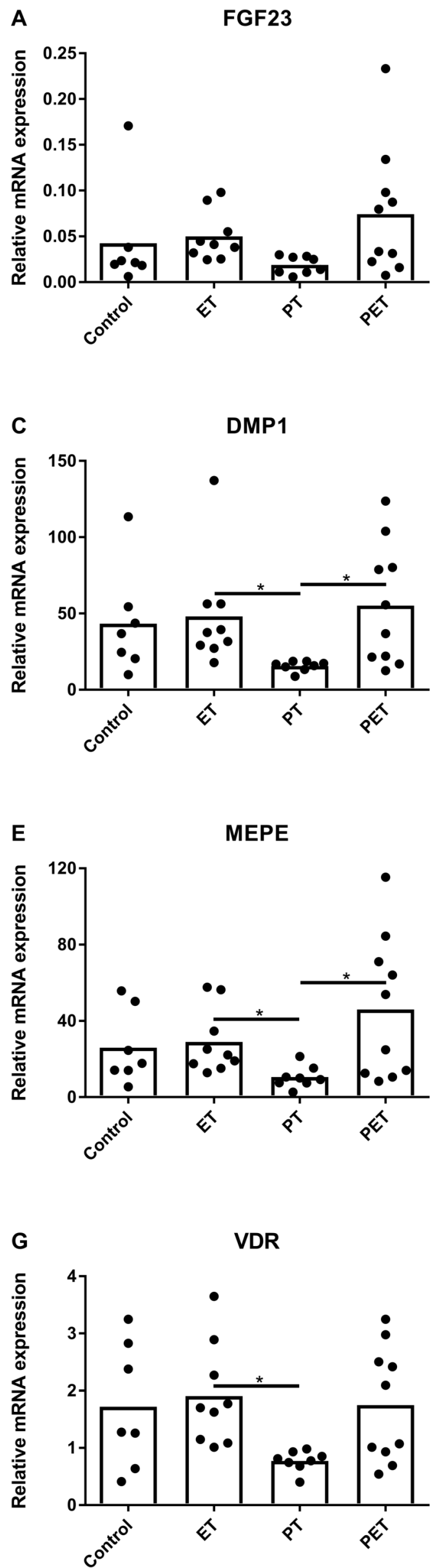

Peerj reviewing PDF | (2018:05:28267:1:0:NEW 10 Nov 2018)
Manuscript to be reviewed

B

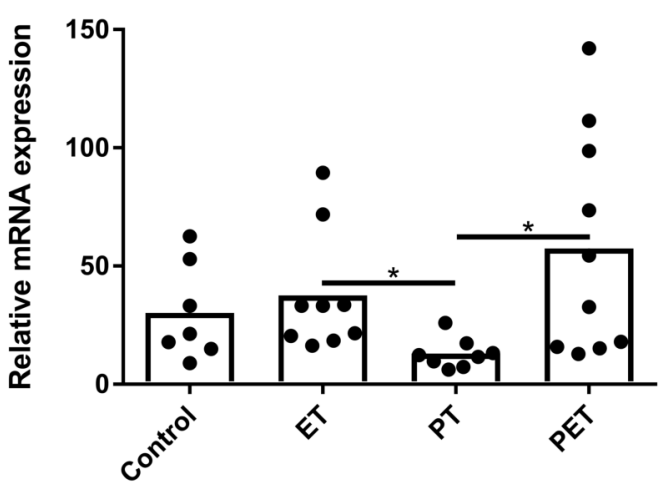

D

PHEX
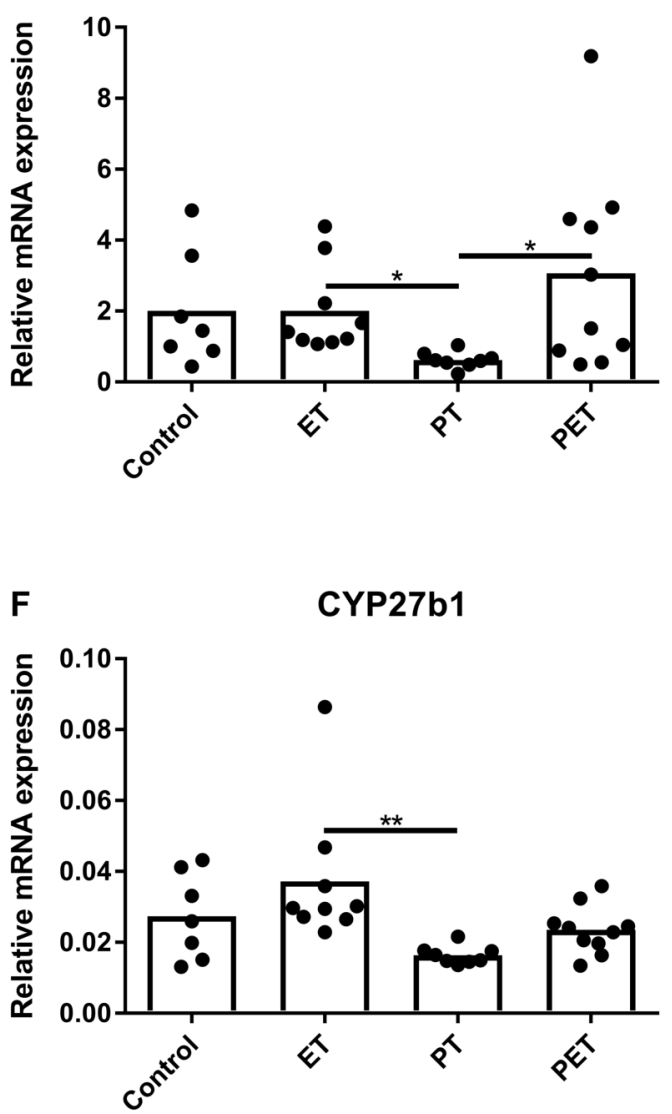


\section{Figure 3 (on next page)}

Effects of different training modalities on mRNA expression in rat kidneys as analysed by qPCR.

Gene expression levels of (A) CYP27b1, (B) CYP24, (C) VDR, (D) NPT2a and (E) NPT2c were measured. Results were normalized for reference genes HPRT and PBGD. Significant difference is indicated as $* p<0.05$.

(ET: endurance training; PT: peak training; PET: combined peak- and endurance training) 
PeerJ

A

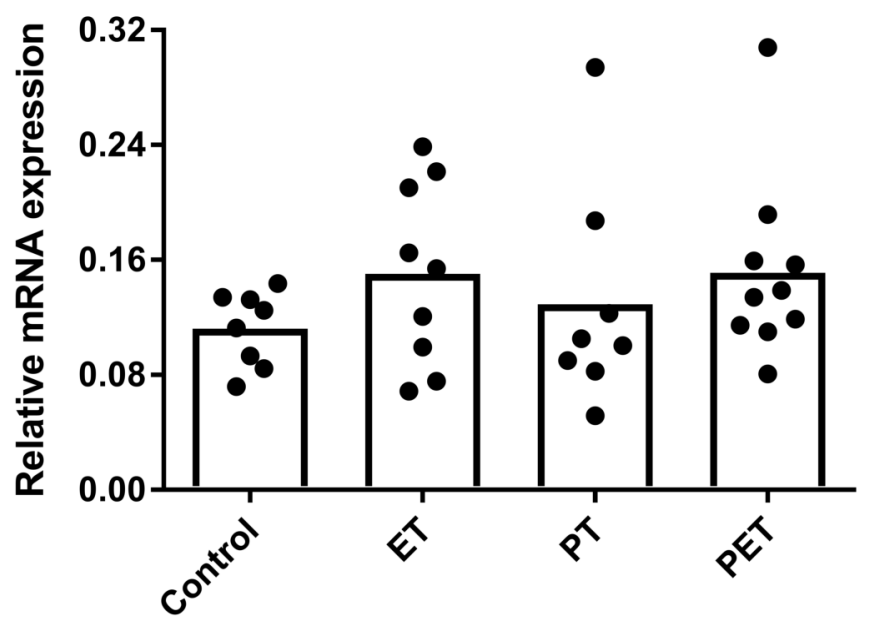

C
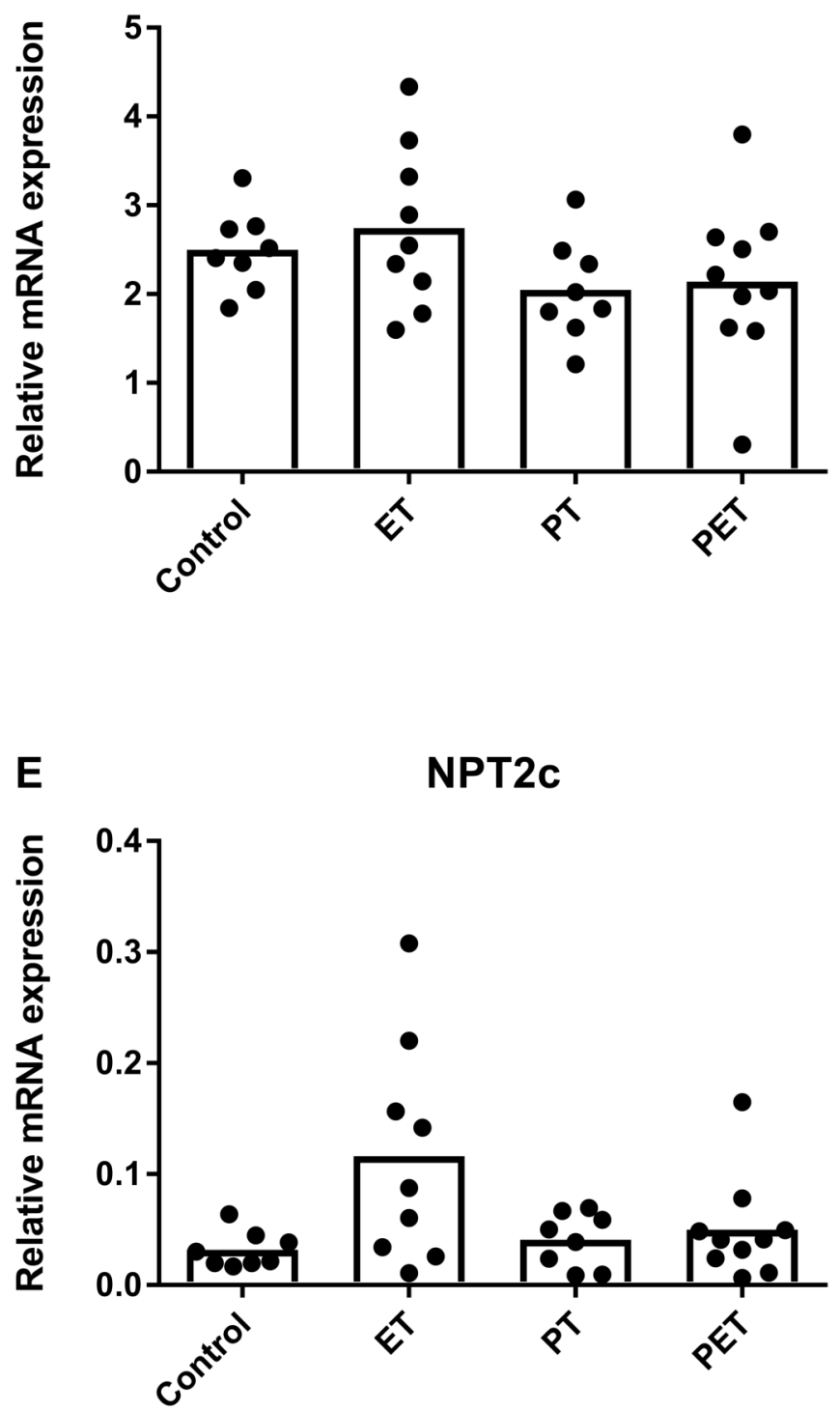

B

CYP24

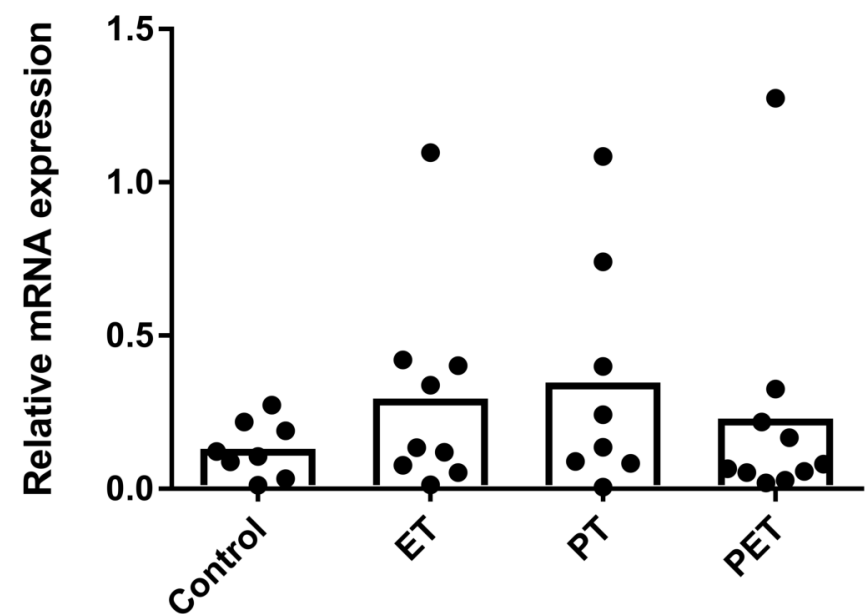

D

NPT2a

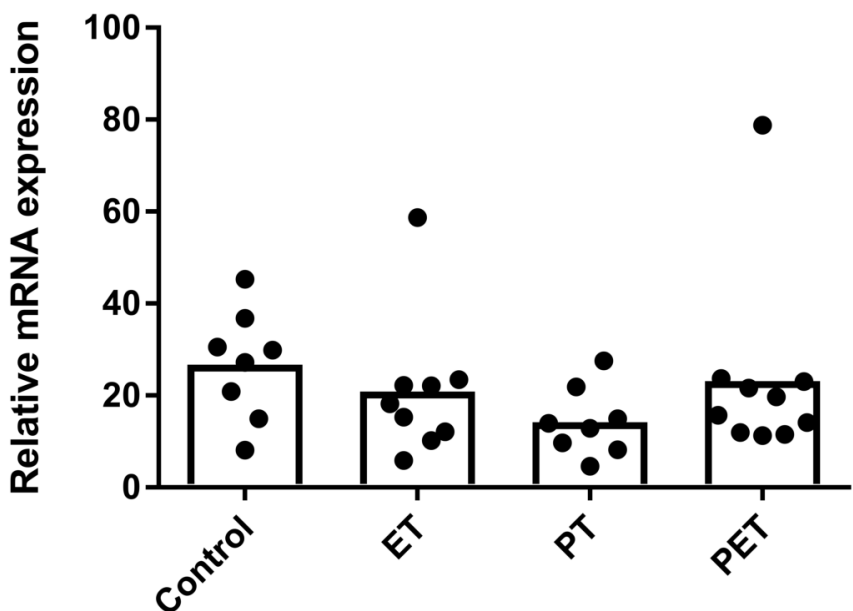




\section{Figure 4 (on next page)}

Effects of different training modalities on serum concentrations of factors involved in phosphate homeostasis.

Serum concentrations of (A) c-terminal FGF23, (B) 25(OH)D3, (C) parathyroid hormone (PTH), (D) calcium and (E) phosphate were assessed. Significant difference is indicated as $* p<0.05$. 

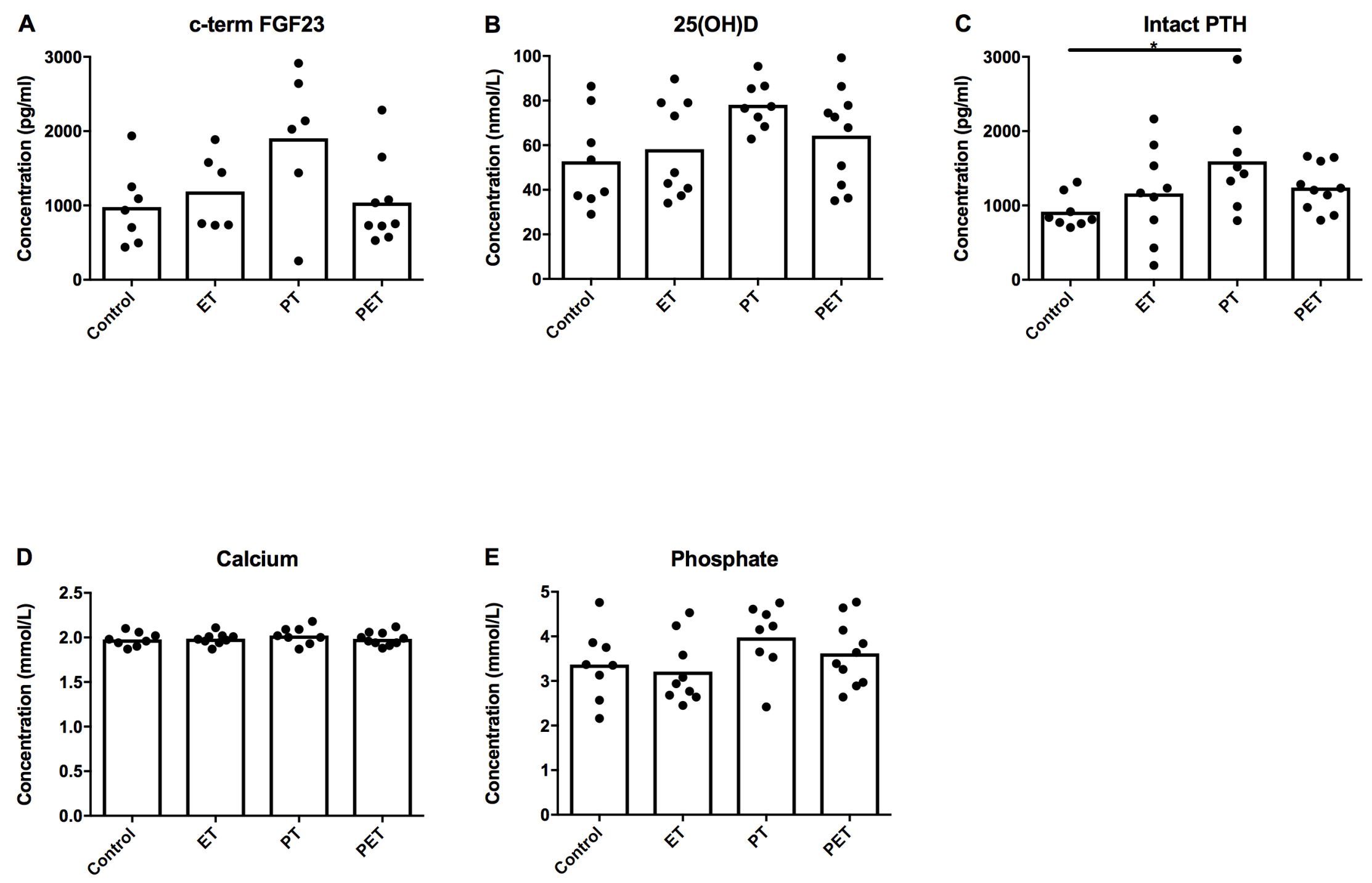


\section{Table $\mathbf{1}$ (on next page)}

Details of primers used for quantitative PCR analysis.

CYP24: 24-hydroxylase; CYP27b1: 1 $\alpha$-hydroxylase; DMP1: dental matrix protein 1; FGF23: fibroblast growth factor 23; HPRT: hypoxanthine phosphoribosyltransferase; MEPE: matrix extracellular phosphoglycoprotein; NPT2a: NaPi-Ila cotransporter; NPT2c: NaPi-Ilc cotransporter; PBGD: porphobilinogendeaminase; PHEX: phosphate-regulating gene with homologies to endopeptidases on the X chromosome; SOST: sclerostin; VDR: vitamin D receptor. 


\section{Table 1.}

PCR primer sequences 5' --> 3'

\begin{tabular}{lll}
\hline \hline Target gene & \multicolumn{1}{c}{ Forward } & \multicolumn{1}{c}{ Reverse } \\
\hline CYP24 & GCT-GAT-GAC-AGA-CGG-TGA-GA & TGT-CGT-GCT-GTT-TCT-TCA-GG \\
\hline CYP27b1 & CCC-GAC-ACA-GAA-ACC-TTC-AT & GGC-AAA-CAT-CTG-ATC-CCA-GT \\
\hline DMP1 & GCG-ACT-CCA-CAG-AGG-ATT-TC & GTC-CCT-CTG-GGC-TAT-CTT-CC \\
\hline HFF23 & GAT-GCT-GGC-TCC-GTA-GTG-AT & CGT-CGT-AGC-CGT-TCT-CTA-GC \\
\hline MEPE & GTG-TCA-TCA-GCG-AAA-GTG-GA & TAC-TGG-CCA-CAT-CAA-CAG-GA \\
\hline NPT2a & AAG-ACA-AGC-CAC-CCT-ACA-CG & CCC-ACT-GGA-TGA-TGA-CTC-ACT \\
\hline NPT2c & AGT-GGC-CAA-TGT-CAT-CCA-GA & AGT-GAT-GGC-TGA-GGT-GAA-CA \\
\hline PBGD & GGT-CAC-CGT-CCT-TGT-ACA-GA & GAC-GCC-CAT-GAT-GAT-AGG-GA \\
\hline PHEX & ATG-TCC-GGT-AAC-GGC-GGC & CAA-GGT-TTT-CAG-CAT-CGC-TAC-CA \\
\hline SOST & CAG-GCA-TCA-CAT-TCA-CCA-AC & GGA-GGA-CTG-TGA-GCA-CCA-AT \\
\hline VDR & CAG-CTC-TCA-CTA-GCC-CCT-TG & GGG-ATG-ATT-TCT-GTG-GCA-TC \\
\hline
\end{tabular}

2

3 